\title{
WHAT DARWIN COULD TEACH US TODAY ABOUT MODERN VACCINES AND RELATED HEALTH POLICIES
}

\author{
G. Corbellini ${ }^{1}$, L. Pani $23,4,5$ \\ ${ }^{1}$ Department of Molecular Medicine, Sapienza University of Rome, Italy \\ ${ }^{2}$ Department of Biomedical, Metabolic and Neural Sciences, University of Modena and Reggio Er. " 'a, ı' 'dena, Italy \\ ${ }^{3}$ Department of Psychiatry and Behavioral Sciences, University of Miami, Miami, I ICA \\ ${ }^{4}$ WCG-VeraSci, Durham, NC, USA \\ ${ }^{5}$ Relmada Therapeutics, FL, USA
}

Like Molière's bourgeois Jourdain, who realized he had been sprakı ð prose for forty years without knowing it, we have been talking about Darwinian evolut.iv.' since the pandemic began and in increasingly stringent ways for the past year, without most if us even realizing it. And yet, the emergence and spread in different epidemiological eco' vstems of new variants of SARSCov-2 were self-evident facts. Facts so clear that even r.ac'eınatics, usually abused to make daily and often disproven predictions of epidemiolog. - $^{-l_{1}{ }^{-h}}$ anges with increase/decrease of cases or deaths, was not needed. The recurring q' 'estiorı are whether the new variants are more efficient in replicating, transmitting, or causin s $u_{i}$ mage to the host, or if they will escape human immune responses by natural infection or hy accine artificial training, changing their antigenic conformations. These are question $\mathrm{p}$ frtaining to Darwinian immunology and vaccinology. The topic also concerns the hot $a^{2}$ ate on the origin of SARS-CoV-2, which at times takes the narrative of a confrontatic. be :ween those who think that natural selection, with favorable conditions at its disposal, a cr $\in$ $\in \mathbb{d} d$ the changes necessary to infect humans, and those who instead think that some sutı...ate of probability of a phenotypic variant (furin cleavage site) is sufficient to provide $a \leqslant n$ t of intelligent design. Darwin today would probably warn us about the risks of vaccina ion strategies that over treats ( $3^{\text {rd }}$ and $4^{\text {th }}$ doses) certain affluent populations without cor sir ring the long-term consequences at the planetary level of leaving vast part of our specie still ilnerable to the combinatorial ability of the Covid-19 virus.

\section{Darwin and Pasteur}

In the tradition of $\epsilon$ 'olı tionary or Darwinian medicine, Darwin is confronted with Pasteur, because the latteı $w^{\prime} \wedge$, along with Claude Bernard and Robert Koch, the lord of the "proximate or immediate cau as of diseases. Such causes can be studied experimentally in the laboratory as in case of nat. ' senic parasites, while Darwin was the master of the "remote or evolutionary causes" of iolugical changes, those that are discovered by studying with observational, experime $11.1 /$ mathematical methods the natural history of living species, i.e. how and why their phen, 'nic ${ }^{+}$aits and underlying genotypes/pools of genes change in the course of descent. Pa-toul's ideas and methods invented scientific medicine and generated the ambition to elimirite or control all microbial causes of disease. Except for the remote causes, those stemming from the fact that human phenotypes develop from a genomic pool selected by evolutionary adaptation by the environment, most of the accumulated "defects and predispositions" could have been arisen at the time to confer an advantage. Thousands of years 
later, those same mutations, now may represent a liability in the changed environmental conditions, to the point that perhaps one day we will be asked (and able?) to correct them only with technologies more advanced than the ones of genomic editing. In short, Pasteur allowed us to imagine and build vaccines or drugs, while Darwin explained, with his theory of nat.ral selection, that not even with the means of the most advanced medicine we would ' $c_{n}^{\prime}$ 'ize the best of all possible worlds.

Moreover, while Darwin could be defined as a layman, who followed the c'e'c'spments of microbiology, Pasteur was a religious conservative, and had no sympath ro ine theory of evolution, which resonated with him as the aberration of believing that $w$ - 2 ascended from monkeys. In the late nineteenth century, some French microbiologist Pasteur was to medicine what Darwin was to natural history, in the - "nst that the techniques aimed at developing transformed pathogenic strains to be used as wr.c.nes applied the same principles as Darwinism or in some ways were artificial selection. Th心 ' $N$ ' As not the case. The idea that guided Pasteur was that the culture medium of micrc rga nisms induced hereditary changes in Lamarckian ways (Darwin too believed in the heritability of acquired traits but not in a drive toward change in an adaptive sense) and caused new sp scies to emerge with attenuated virulence. Ready for vaccines. The French microbi, lo, ist thought that immunity had no independent physiological basis but was due to the mert repletion of nutritional factors in the host, after the pathogen had lodged there leaving th. host alive.

The modern concept of vaccination and its shept er $\mathrm{d}$ ?ffect, is not directly related to Darwinism, because it is inscribed in an idea of immunı,; as a passive phenomenon and in some late nineteenth-century physiological models or II.mune response that sensed the active and dynamic nature of the phenomenon. We has $h$ wait until the fifties to begin to understand and then demonstrate that immunity ins ${ }^{+} \mathbf{t a}^{\prime} \lambda$ depends on a complex genetic-molecular architecture, which governs the dynar ic, ytokines-mediated, interactions of cell populations with different roles and where the $s^{\prime}$, eci ${ }^{+}$Icity of responses is then governed by clonal selection. An idea, that of clonal selection, tr + , ank Mcfarlane Burnet, in the late fifties, directly linked to the Darwinian idea of natural seltec tir $n$.

Vaccine makers have long ' $\sim$ ? $n$ artisans, rather than scientists, and they did not design them based on a controlled hrov' 'sge of immunity but arrived the result by trial and error. The logic of invention, as it happeris for many innovations and according to Karl Popper also for scientific theories, is still jartly evolutionary.

\section{Origin and tec:"nol .gical evolution of vaccines}

Vaccines are $\sim n$ invention of human ingenuity and descend from observations and magical thoughts. ' h'ıcydides was the first to observe during the so-called Athens' plague of 430 b.C. that $p \in \sim$ '? who recovered from an infectious disease did not get sick a second time (immunity acqu reu naturally). Likely, the phenomenon had already been reported, but the context of the "plague of Athens", where people were crowded and the disease had a rapid clinical course as being lethal or healing, facilitated its observation and description. We can also imagine that since antiquity it was realized that milder forms of a specific contagious diseases protected 
from more severe forms. Alastrim, due to the variola minor species, would immunize against classical human smallpox, caused by variola major, with a mortality of about $1 \%$, versus $30 \%$, respectively. The widely held homeopathic magical principle that "like cures like" (similia similibus curantur) was probably an early intuitive interpretation for such naturally c'ise 'ed effects. Artificial immunization, using Alastrim, or variolation, according to Joseph ive viham was practiced in China around the year 1000, and in 1720-22 came to Europe. It wa-s.'slanted by Jenner's vaccinia, the cow's adapted pox virus, in 1796 (banned in the UK .. 18-i0) using cowpox.

The evolution of vaccines and technologies is a well-known story that ha $1, \sim 1$ jgic or finalistic sense. Precisely like any evolutionary process. After Jenner and the smi "lpux vaccine, which protected against human smallpox by cross immunity, the first $1 \mathrm{~m}_{\mathrm{c}} \mathrm{n}$ vaccine with live attenuated agents (against rabies) came in 1885. Both these vaccint - ware developed before the identification of the causative agent of the disease. They were $v$ or $y$ "dirty" vaccines, in the sense that they could transmit other human or animal pathoger. or : ause adverse reactions as well as the were used without safety and efficacy controls. 3ack 1 , those times, not too long ago, the political, social and health expectations and policiec $v$ vre very different.

Since the first typhoid vaccine in 1896, the discovery of the causative agent has always preceded the creation of the vaccine, with varying 1 nescales, ranging from almost a century for the meningococcal meningitis (1887-1970/5) ?nd Haemophilus influenzae (1892-1985) vaccines, to a few months as in the case of the 19',7 ir fluenzas' vaccine or SARS-CoV-2 vaccine. Social pressures due to the impact of the cine, technological availability and biological characteristics of the pathogen are the valables that influence vaccine research and development. The first inactivated vaccin - a er the one against rabies, were against plague, cholera, and typhoid fever, while in the $t$ 'er ies toxoids (which teach the immune system to recognize bacterial toxins) against di hth ?ria and tetanus entered the field, followed twenty years later by the first polysaccha' de vaccine against pneumococcus. In the sixties, after a series of live attenuated vaccines $:$ iı , oreat health impact (polio, measles, mumps, and rubella), came the first vaccine made up 0 s sbunits (molecular components of the pathogen), against influenza and other viral it ${ }^{\text {f }}$ ections, among which a few against SARS-Cov-2 have been approved in Western and $\mathrm{ra}_{-}{ }^{+} \boldsymbol{e}_{\mathrm{I}} \mathrm{n}$ countries. In the mid-1980s recombinant DNA technology was used for the hepa $i^{+}{ }^{i}$ ? ? vaccine, and subsequent recombinant vaccines included those carried by viral vectors : $:$ ucr, as the adenovirus used against SARS-CoV-2). In the same decade, conjugated polysacc lariłe-based vaccines (anti- $\mathrm{H}$. influenzae $\mathrm{b}$, anti-meningococcal $\mathrm{C}$, antimeningococcal AS' $N$;, entered the scene. The rotavirus vaccine was the first vaccine genetically reas?or ier. to enrich the antigen profile. Today we have the first approved nuclide acid vaccines, : ’am iy mRna vaccines against Covid-19.

\section{Uncertai.1 ;0 jial fortunes of vaccinations}

Vacirite have improved dramatically in terms of efficacy and particularly safety as their approval process has gone through clinical trials since the 1960s. Adverse reactions are now rarer, less severe or more predictable, and incidents due to recovery of virulence from live attenuated vaccines with fatal outcomes less likely. Why is it then, if they are so much safer, 
that prejudice against vaccinations is so persistent or growing? This is normal since we cannot change our perception of risk based on correct information vs. fake news alone. Human decision-making and judgment depend on or/and are controlled by heuristics biases that were acquired by natural selection to survive in EEA. In order to choose and decide, i su me situations, we tend to ignore true information and prefer false ones which is a consf yu ? re of Darwinian evolution.

It does not appear that variolation caused a rejection in Asian and Africis $n$ o vieties that practiced it, while when it arrived in Europe and North America in 17-1-2.2 quite a few theologians, doctors and Enlightenment philosophers opposed it. Amoı $t_{1}$ or.ı, Jean Jacques Rousseau and Immanuel Kant on grounds of unnaturalness and ethics. - mallpox vaccination was also accepted in the compulsory form in several continental cou ${ }^{+}{ }^{+}$ies, less democratic, but not in England invested by liberal ideas. For over half a century, vaccı, ?tı n campaigns in Britan had to face with social protests, which depicted propagandistic. $1 \%$, the inoculation as an interference in personal freedom and a physically disgusting a + . a source of threat to human life. The rejection or hesitation towards vaccination, which could ainost be understood in the nineteenth century since the material and the ways of vaccina ${ }^{\prime}$. nn were quite disgusting, has reemerged in recent decades with preponderance throu , hc ' $\mathrm{t} t \mathrm{t}$ ie West, for psychological and, in part, social and political reasons that probably remain thı -ame.

Many explanations have been given to explain the c ver sion/hesitancy to vaccination, some of which invoke the fact that our minds have not eı o' $\mathrm{s}$ d to trust people we don't know (doctors, or - much less - an abstract entity like the St. ${ }^{2}{ }^{\circ}$ ) vvnen they tell us that there might be some minimal risk in getting vaccinated. If we do $r$ ot - +udy and understand statistics, which we were not taught by natural selection or educrtin, in the Pleistocene, we tend to estimate risks incorrectly. Moreover, propaganda agair - $\mathrm{v}$ accines still recruits the emotion of disgust as a form of contamination; disgust that a : co znitive psychologists show served evolutionarily to defend us from parasites, by nowad iys ivoks to associate vaccines with a danger. Paradoxically, the disappearance of parasites as $\eta \epsilon_{1}$, asive threats has allowed vaccinations, which introduce something foreign into healthy r er,ple and imply a belief on trust that they are safe and protective, to be perceived c dangerous, that is, as polluting practices. Perhaps the best way to counter anti-vaccinism, ic; rast in case of uneducated or emotionally orientated people, is to

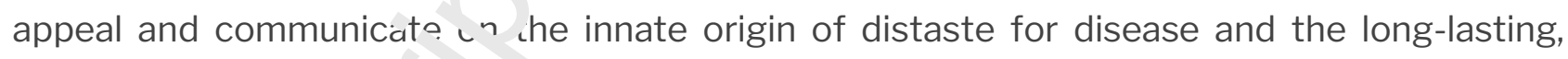
sometime disfiguring a: $d$ le linar effects that infections cause.

\section{Vaccines and mici ubi al evolution}

Since spring oi U 21, the question has been whether and when SARS-Cov-2 will attenuate its contagiou $n \in$ : $s$ and become symbiotic with humans, i.e., whether it will evolve into a run-of-themill cold $j i$ us.. No one can say, although it is possible and some of the recent variants like the Omicrc n -eem to suggest this may be happening. But it is too early to call. One of the cha ${ }^{\prime}$ cu. istics of Darwinian phenomena is that the variations that change the phenotype of an organism like a virus, do not arise with the purpose to make it "better" or optimize it, for becoming more contagious, virulent, pathogenic, etc. or all the contrary. Mutations emerge at 
random, and the fact that they exhibit and preserve specific phenotypic traits depends on the replicative advantage (natural selection) in the environment where they arise. Period.

Pandemic evolution will proceed its own way, in defiance of the human presumption that we can make predictions or know what should be done to bring it under control. This doesn't m' an 'hat nothing should be done, but that perhaps we should also think about maintaining the $\mu_{1}$ ' cuiples $^{-}$ and values we foster in our societies, and not put them at risk to chase controlling $\sim \boldsymbol{v} \cdot$, that is much more efficient than us in its "simple" diffusion strategies. The virus, multiply „ ` ir , different ecological contexts (UK, Brazil, South Africa, India, immunodepressed patier is , hildren, etc.) and thus facing different selective pressures, emerges in the form of diffeier $\therefore$ variants. We certainly do not know them all, since the number of sequenced genomes at 'hr planetary level is impressive but also negligible.

Microbiologists and virologists had predicted since the beginniric 0 , this pandemic that mutations would come, even though, and it still is so, we can't exac ' 'v anticipate where on the planet they will emerge, and we don't understand precisely whi $h \mathrm{p}$ rtions of the genetic code of Sars-Cov-2 will be mutated. What we know is that every tirne a $v_{1}$ ' 1 s replicates to give rise to a copy of itself it can make an error. At the peak of the infec in a human carry from 1 to 100 billion viral particles all potentially capable of generate aln ost as many mutated variants. In the case of coronaviruses this happens more frequently beca 'se these viral families replicate their genetic material in a "sloppier" way than humans and animals or even than some other microorganisms such as those DNA based. Corona iru es contain single-stranded ribonucleic acid (Rna) and evolutionarily enzymes that makf $r$ i/ copies of Rna are more prone to errors. Even though coronaviruses do not produce is many mutations as other Rna viruses, e.g. influenza viruses, because their correctior eı. rymes repair transcriptional errors, they still produce a significant quota of mutations. Farc ronaviruses, it is estimated that an error occurs for every one million replicated units or, $t$. ' a ' easonable estimate, that each infected individual carries between 1,000 to 100,000 va ian s (often the same variant) to be made available for subsequent environmental selectio $\mathrm{I}$.

The question is therefore wheth $\epsilon^{-}$the new harmful variants will be blocked by the approved vaccines or by those coming and whether the vaccines, as will be the case for some, which do not have complete efficacy, $v^{\text {: }}$ " I avor the evolution of strains that survive, that is, whether they will become more benign. $\iota^{-}$- shether there will be a selection in favor of virulence and most importantly lethality. Tr f first answer is easy: it depends on how the variants are changed at the level of epitopes (ant gers) recognized by the immune system. This is not a serious problem: if the current vaccin s ive efficacy, the information to be sent to the cells can be changed to make them expreso $n$ ', w immunogenic epitopes. The platforms set up over the past year by the companies tha ${ }^{+}$me ire the vaccines, in particular those based on $\mathrm{mRna}$, are flexible enough to

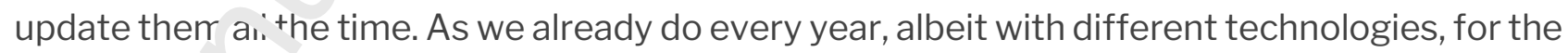
flu vaccine

The $s \in \cdot u$ d question does not have a precise answer. We have long wondered if there is resı +alı a to vaccines, as to drugs, and if so, how it evolves. In fact, it is not frequent. Resistance to drugs, for example antibiotics or anticancer drugs, typically emerges soon after the introduction of a new active ingredient because of the selection of variants (bacterial or tumor cells for example) that escape the action of drug treatment. 


\section{But full vaccine resistance has rarely emerged. Why?}

Vaccines tend to work in a prophylactic way, while drugs are therapies, i.e. optimal vac' ine_do not allow replication and consequent growth of pathogen populations. No repl'va' 'Or, no evolution. In addition, vaccines tend to induce immune responses against multipie ta. ar, ts on a pathogen, while drugs hit very few targets. As a result, pathogen populations ontrate less variation for vaccine resistance than they do for drug resistance, and sfie; ion has less opportunity to act on that variation.

So far, we have been fortunate in the sense that the efficacy of most $v^{-}$.cines used has not been completely compromised by evolution of the targets. Smallpox : cclı ation eradicated the human smallpox virus without giving it any possibility to evolve. The : ${ }^{\prime} c$ ine was also the best combination of antigenic stimuli, that is, was the closest design h ratural human smallpox, which had no animal reservoirs. But neither did any strain emerg fro $n$ the measles, rubella, etc. virus families that could circumvent vaccine-induced imm'nity. The diphtheria vaccination apparently favored the more benign strain of bacteria. W/t to know of a few exceptions, however.

\section{Darwinian Vaccinology}

In 2001, the molecular ecologist Andrew Rt $\mathcal{f}$ published in Nature a mathematical model showing that the imperfect (very imperfec $i$ ) $v$ ccines then being tested against falciparum malaria, risked increasing the virulence of tho $p_{n}$ otozoan. In the two decades that followed, that mathematical model has found several rea-world applications, explaining for example the increase in virulence favored by vaccil atic $n$ in the case of Marek's disease of chickens, due to a herpesvirus and causing losses o about 2 billion USD per year for the poultry industry. The vaccine works as an anti-disear? p.uphylaxis, and benefits the immediate health of the chickens, but it favors the evolut, $\mathrm{c}$ r, $f$ more virulent strains over time and implies a run-up with gradually adjusted vaccines ' 'iere are three Marek vaccines developed so far, since 1970). Two similar cases are known in 4 . ? world of animal breeding, involving some infections in farmed

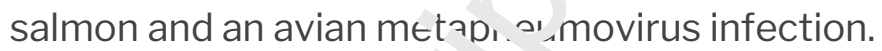

Mathematical model; susgest that vaccines that are protective for individuals in clinical trials may nevertheless ge benefits of diseast-fic,hting or imperfect vaccines mask an increased risk of evolution of more virulent strain - T,e acellular pertussis vaccine, compared with the earlier vaccine that contained $t^{\prime} ı$ kllled bacterium, confers shorter immunity, so there have been several recurrencac c: the disease, and the cause has probably been evolutionary opportunity. Acellular is preforr 2 k ecause it has no side effects. Several vaccine-resistant strains of hepatitis B virus have $b_{1} \epsilon_{1}$ detected and their health impact is being studied. The history of the Prevnar series of vir.cin as $(7,13)$ against pneumonia, otitis, meningitis, etc. from S. pneumonia in children and adults is an interesting case, because there are 90 distinct serotypes of the bacterium and the early vaccines intercepted 7 and 13 . Prevnar 7 had reduced cases and deaths, but it had led to an evolutionary restructuring of the bacterial population, which seems to be happening with 13 
as well. The restructuring is continually ongoing, and the evolution is aided by the fact that vaccination is done primarily in the United States but not in Europe and other parts of the world.

Because of microbial evolution, some pathogens for human diseases such as naluria, trypanosomiasis, AIDS, etc., use different strategies to change recognizable a.tigenic structures or do not show up at all to the immune system. These are the cases wnor of iective vaccines are difficult or impossible to develop. In agricultural contexts, where i., mediate and economically satisfactory solutions are sought, several animal vaccines are 1 'termined by microbial evolution. The virulence of a pathogen is directly related to re'sicái.ion and more pathogen also equals more disease, so every effort should be made to pro ${ }^{\prime}$ 'ir e vaccines that nullify replication, and thus transmission.

\section{Darwin versus Covid-19}

If the SARS-CoV-2 virus were to evolve in response to an anti COV 2 vaccine, it would probably adopt the strategy of the influenza virus, i.e. continually mitais the surface molecules against which the antibodies work just as long as the epitopes re nair the same. The virus would soon escape detection and it would be a matter of periodicálı, 'ıpdating, as with influenza, the antiCovid vaccine. Evolution could lead the virus to bec ne, under vaccine pressure, invisible and semi-innocuous, perhaps reproducing slowly or hidi. 'o ir organs where immunity is less active.

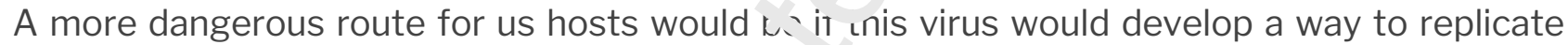
more rapidly than the immunity generated I,y ine vaccine. Another strategy would be for the virus to decide to target the immune system $a_{1}$, directly dampen vaccine-induced immunity as in the case of rabbit myxoma. Andrew Rec ' ar d his group believes that the truly evolution-proof vaccines are those that are very effect 've , $t$ fully suppressing viral replication, so they stop any further transmission.

Indeed, the ideal vaccine involves: no replication, no transmission and thus no evolution. Furthermore, to be evolu: in-proof, a vaccine must activate immune responses that simultaneously attack diffe.c. 't parts of the pathogen. It is normal for some part of the virus to mutate and evade the tarot: r, ut if many sites are recognized simultaneously, immune evasion requires the highly : nlikely possibility that many separate evasive mutations occur simultaneously. In la soratury SARS-CoV-2 rapidly developed resistance toward monoclonal antibodies, but strı ogu to develop resistance against a cocktail of antibodies, targeted toward multiple and differ ent sites. Evolution-proof vaccines protect against all circulating strains, so that no other $v$ riar : can fill the void when competitors are eliminated.

According tc Read's model before finding out how many have the evolution-proof chararte.is tir,s, a little extra effort could be made during trials to find out if a vaccine will be evolutı, nroof. By running swabs on people who have received the experimental vaccine, one cour ${ }^{\top} \mathrm{uc}$. ermine to what extent viral levels are suppressed and by analyzing the genome of the viruses in the vaccinated people, one could check to see if an evolutionary escape is taking place. Finally, by taking blood from the vaccinated, one could calculate in the laboratory how many sites of the virus are recognized by vaccine-induced immunity. 
Settling for partial or temporary relief as an effect of a vaccination strategy is evolutionary unwise, because while benefits are observed for individuals, at the population level and over the long-term people remain vulnerable. And the problem is only displaced, until the next v riar. . or the next pandemic emerges.

\section{Disclosures}

LP in the past two years has acted as a scientific consultant for Bo shri ger Ingelheim International GmbH, Germany; Compass Pathways, UK; EDRA-LSWh PL'.rlishing Company, Italy; Inpeco SA, Switzerland; Johnson \& Johnson USA; Novartis-Genє I, r rapies, Switzerland; Sanofi-Aventis-Genzyme, France and USA; Relmada Therapeutics, U`^., WCCT World-Wide Clinical Trials, USA; WCG-VeraSci, USA. He has never consultec' on vaccines research and development.

GC has no conflict of interest to declare.

\section{Essential References}

AA.VV. (2020), Vaccines, Nature Milestones, Nov_-1 Juer 2020.

Alizon S, Méthot P-O (2018), Reconciling Pas `uı and Darwin to control infectious diseases. PLoS Biol 16(1): e2003815

Corbellini G., Evolution Based Medicine ( $(-E . \downarrow)$ in Epistemological Framework For Thinking of, and Dealing with the so Called Crisis nt ' 1r dicine. Medicina nei secoli 2008; 20 (1), 115-139

Geoghegan JL, Holmes EC. The pr; 10 rsnomics of evolving virus virulence. Nature Reviews Genetics, 2018; 19(12), 756-769

Kennedy DA, Read AF (2018) WI y th e evolution of vaccine resistance is less of a concern than the evolution of drug resista.' 'e. Proc Natl Acad Sci USA. 2018; 115(51):12878-12886.

Kennedy DA, Read AF (207), Vhy does drug resistance readily evolve but vaccine resistance does not? Proc Royal Soc B. : . 117; 284:20162562.

Kennedy DA, Read AF '2 ᄂ? O) Monitor for COVID-19 vaccine resistance evolution during clinical trials. PLoS Biol 18(11, e:001000.

Luz PM, Brown $\mathrm{H}^{\prime}$, , str uchiner CJ (2019), Disgust as an emotional driver of vaccine attitudes and uptake? A mec" "ztic $ı$ analysis, Epidemiology \& Infection; 147: e182.

Moulin AM ‘'OO J), Darwinisme et pastorisme, https://hal.archives-ouvertes.fr/hal-00434433 